\title{
Polymicrobial brain abscess involving Haemophilus paraphrophilus and Actinomyces odontolyticus
}

\author{
AJH Simpson, SS Das, IJ Mitchelmore
}

\begin{abstract}
Summary
A case of brain abscess involving Haemophilus paraphrophilus and Actinomyces odontolyticus is presented. This combination of organisms has not previously been described. All brain abscess specimens should routinely be processed rapidly and cultured for a prolonged period to ensure recovery of fastidious organisms which may have implications for antibiotic therapy.
\end{abstract}

Keywords: brain abscess, Haemophilus paraphrophilus, Actinomyces odontolyticus

We report a case of brain abscess involving Haemophilus paraphrophilus and Actinomyces odontolyticus in addition to several anaerobes. These two organisms are rarely isolated from brain abscesses, and have not previously been described in combination.

\section{Case report}

A 66-year-old woman was admitted with a 10 day history of headache, drowsiness and blurred vision. She had an episode of mild toothache one month previously, which resolved without intervention. There was no history of sinusitis, otitis or lower respiratory tract infection.

On examination she was pyrexial $38.6^{\circ} \mathrm{C}$, drowsy but fully orientated, without neck stiffness. She had an ejection systolic murmur, and a left homonymous hemianopia. There was a faint left hemiparesis, with an equivocal left plantar reflex. The diagnosis was of a right occipital space-occupying lesion. She had a peripheral white cell count of $9.9 \times 10^{9} / 1$. A computed tomography (CT) scan demonstrated an irregular mass in the right occipital lobe, consistent with a cystic malignant glioma. The patient had been started on intravenous benzylpenicillin, flucloxacillin, gentamicin and metronidazole, and was also taking dexamethasone, $4 \mathrm{mg}$ 6 hourly. At craniotomy an abscess was found. This was drained and the capsule excised.

Gram stain of pus showed numerous white cells, Gram-positive cocci and small Gramnegative rods. Gas-liquid chromatographic (GLC) examination of the pus showed butyric, acetic and propionic acid peaks, indicative of the presence of anaerobes. Antibiotic therapy was changed to benzylpenicillin, cefotaxime and metronidazole. Histological examination revealed no evidence of malignancy. The patient made a good postoperative recovery, and an infective source was sought. Blood cultures remained sterile. An echocardiogram showed mild mitral regurgitation only. Dental examination revealed a small granuloma below a pre-molar tooth.

The speciment was incubated on blood, chocolate and CLED agar at $37^{\circ} \mathrm{C}$ in air plus $5 \% \mathrm{CO}_{2}$, and on blood and neomycin agar anaerobically ( $80 \%$ nitrogen, $10 \%$ hydrogen and $10 \% \mathrm{CO}_{2}$ ). Prolonged culture (14 days) revealed Haemophilus paraphrophilus, Peptostreptococcus micros, Fusobacterium nucleatum, Actinomyces odontolyticus and another unidentified anaerobic Gram-negative rod, all in heavy growth. In addition, $P$ magnus was recovered from an enrichment broth. The $H$ paraphrophilus grew on chocolate agar only, was V-factor dependent and fermented glucose and sucrose. It was weakly oxidase positive, catalase and indole negative, urease and ornithine decarboxylase negative but $\beta$-galactosidase and gammaglutamyl transferase positive. It was susceptible to ampicillin, cefotaxime and chloramphenicol. The $A$ odontolyticus was nonpigmented, was catalase, indole and aesculin negative and fermented glucose, sucrose, ribose and xylose. It produced acetic, lactic and succinic fatty acid peaks on GLC. It was susceptible to penicillin and chloramphenicol.

The patient was discharged after three weeks, to continue with oral amoxycillin for three months, and metronidazole for three weeks. She remains well, and her visual fields are improving.

\section{Discussion}

Brain abscesses are often polymicrobial, especially if they arise from a dental source as seems likely in this case. ${ }^{1}$ Anaerobes, often multiple, especially Peptostreptococcus and Bacteroides (including Porphyromonas and Prevotella) species, are commonly isolated from brain abscess and are consistent with a dental or otogenic origin. ${ }^{2,3}$ However, the presence of $A$ odontolyticus and $H$ paraphrophilus made it more likely that dentogingival sepsis was the primary focus.

$H$ paraphrophilus has very rarely been identified as a human pathogen since its description in $1968,{ }^{4}$ with isolated reports of brain abscess. It is $\mathrm{CO}_{2^{-}}$and $\mathrm{V}$-dependent, oxidase positive and catalase negative. The $\mathrm{V}$-factor requirement may limit its growth on primary plates unless these contain blood or have been supplemented. It is not a difficult organism to isolate provided suitable media, such as cho- 


\begin{tabular}{|c|c|}
\hline \multicolumn{2}{|c|}{ Microbial causes of brain abscess ${ }^{1-3,9}$} \\
\hline $\begin{array}{l}\text { Species } \\
\text { Streptococcus spp, particularly } \\
\text { S milleri group } \\
\text { Anaerobic Gram-negative rods } \\
\text { (Bacteroides, Prevotella and } \\
\text { Porphyromonas spp) } \\
\text { Peptostreptococcus spp, particularly } \\
\text { P micros } \\
\text { Staphylococcus aureus } \\
\text { Enterobacteriaciae (eg E coli) } \\
\text { Fusobacterium spp } \\
\text { Haemophilus spp, Actinomyces spp, } \\
\text { Mycobacterium tuberculosis, fungi } \\
\text { (Aspergillus and Candida spp), } \\
\text { Toxoplasma gondii, helminths (eg, } \\
\text { Strongyloides stercoralis) }\end{array}$ & $\begin{array}{l}20-50 \\
10-47 \\
10-30 \\
10-33 \\
0-10 \\
<1\end{array}$ \\
\hline
\end{tabular}

\begin{tabular}{|l|}
\hline Antibiotic regimens for empiric \\
therapy of brain abscess \\
\hline - benzylpenicillin+chloramphenicol+ \\
metronidazole \\
- chloramphenicol+metronidazole \\
- cefotaxime+metronidazole $( \pm$ benzylpenicillin $)$ \\
\hline
\end{tabular}

\begin{tabular}{|l|}
\hline Summary/learning points \\
\hline - brain abscesses require surgical drainage where \\
possible \\
- close and careful monitoring is required to \\
ensure a successful outcome \\
- brain abscesses are commonly polymicrobial in \\
aetiology \\
- isolation and identification of all organisms \\
from brain abscess specimens is vital to allow \\
rational decision-making about the optimal \\
antibiotic therapy \\
- prolonged incubation of specimens under a \\
wide range of cultural conditions is necessary \\
to ensure recovery of all organisms \\
- brain abscesses may follow minor dental or ear \\
infections
\end{tabular}

colate agar, are inoculated, and provided incubation occurs in a $\mathrm{CO}_{2}$-containing atmosphere. However, mis-identification may occur, possibly because the organism may become less fastidious on subculture. It is likely that some isolates have been misidentified as the more commonly isolated organism $H$ parainfluenzae, which is also $\mathrm{V}$ -

1 Wispelwey B, Scheld WM. Brain abscess. In: Mandell GL Bennett JE, Dolin R, eds. Mandell, Douglas and Bennett's Bennett JE, Dolin R, eds. Mandell, Douglas and Bennett's Principles and practice of infectious diseases, 4th

2 Richards J, Sisson PR, Hickman JE, Ingham HR, Selkon JB. Microbiology, chemotherapy and mortality of brain abscess in Newcastle-upon-Tyne between 1979 and 1988. Scand $\mathcal{f}$ Infect Dis 1990; 22: 511-8.

3 Brook I. Bacteriology of brain abscess in children. $f$ Neurosurg 1981; 54: 484-8.

4 Zinneman K, Rogers KB, Frazer J, Boyce JMH. A new Vdependent Haemophilus species preferring increased $\mathrm{CO}_{2}$ tension for growth and named Haemophilus paraphrophilu nov.sp. F Pathol Bacteriol 1968; 96: 413-9.

5 Krishna L, Slizowski WJ, Katsetos CD et al. Abnormal intracerebral thallium localization in a bacterial brain abscess. F Nucl Med 1992; 33: 2017-9. factor dependent, so the importance of this organism may be understated. It may occassionally be confused with $H$ aphrophilus or Actinobacillus actinomycetemcomitans which, like $H$ paraphrophilus, are normal human oral commensals. A polymicrobial abscess involving $H$ aphrophilus and $A$ odontolyticus following a molar abscess has been reported. ${ }^{5}$ However, to our knowledge, no reports of mixed infection involving both $H$ paraphrophilus and $A$ odontolyticus have been published.

$A$ odontolyticus is also rarely described as a human pathogen. The organism is found in the human oropharynx as a commensal and it may be associated with dental plaque production. It is a Gram-positive pleomorphic rod which grows slowly, both aerobically and anaerobically in added $\mathrm{CO}_{2}$. There are a few reports of systemic actinomycosis due to this organism ${ }^{6}$ and several linking $A$ odontolyticus with brain abscess.,7 Actinomycotic infection is often polymicrobial, commonly with anaerobic organisms and is probably a synergistic process. ${ }^{8}$ Actinomycosis requires prolonged penicillin therapy. However, the optimum dose and duration of treatment is unknown.

For brain abscess, early surgical drainage is essential, together with antibiotic therapy to ensure successful cure. Patients require careful monitoring and follow-up. Isolation of fastidious or anaerobic organisms requires rapid processing of clinical specimens and prolonged culture for adequate recovery, together with detailed studies to identify isolates. Early reports of brain abscess bacteriology demonstrated a number of 'sterile' abscesses which were probably due to inadequate specimen handling and processing. ${ }^{9}$ These specimens should undergo lengthy incubation as otherwise important isolates may be missed, such as the $A$ odontolyticus in this case, which may influence antibiotic therapy.

Ideally, any primary source such as a dental abscess requires removal to prevent recurrence, but it was felt that the possible small dental abscess had been adequately treated with antibiotics. However, this case emphasises the point that early collaboration with a dental surgeon is also important in the successful management of a patient with brain abscess.

We thank Mr H Sabin for permission to report this case. We also thank the PHLS Anaerobe Reference Unit, Cardiff and the PHLS Haemophilus Reference Unit, Oxford, for their assistance in identification of these isolates.

6 Peloux Y, Raoult D, Chardon H, Escarguel JP. Actinomyces odontolyticus infections: review of six patients. $\dot{F}$ Infect 1985 odontolyticus

7 Hutton RM, Behrens RH. Actinomyces odontolyticus as a cause of brain abscess. $\mathcal{F}$ Infect $1979 ; 1: 195-7$.

8 Philips JE. Actinomycosis, actinobacillosis and related diseases. In: Parker MT, Collier LH, eds. Topley and Wilson's Principles of bacteriology, virology and immunity, 8th edn, vol 3. Bacterial diseases. London: Edward Arnold, 1990; pp. $31-43$

9 de Louvois J. The bacteriology and chemotherapy of brain abscess. F Antimicrob Chemother 1978; 4: 395-413. 